\title{
WORKPLANS: Workshop on Planetary Nebula Observations
}

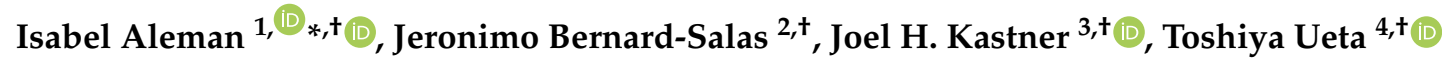 \\ and Eva Villaver $5,+(\mathbb{D}$ \\ 1 Instituto de Física e Química, Universidade Federal de Itajubá, Av. BPS 1303, Pinheirinho, \\ Itajubá 37500-903, Brazil \\ 2 ACRI-ST, 260 Route du Pin Montard, 06904 Sophia-Antipolis, France; jeronimo.bernard-salas@acri-st.fr \\ 3 Center for Imaging Science, School of Physics and Astronomy, and Laboratory for Multiwavelength \\ Astrophysics, Rochester Institute of Technology, 54 Lomb Memorial Drive, Rochester, NY 14623, USA; \\ jhk@cis.rit.edu \\ 4 Department of Physics \& Astronomy, University of Denver, 2112 E. Wesley Ave., Denver, CO 80208, USA; \\ Toshiya.Ueta@du.edu \\ 5 Departamento de Física Teórica, Universidad Autóonoma de Madrid, 28049 Madrid, Spain; \\ eva.villaver@uam.es \\ * Correspondence: bebel.aleman@gmail.com \\ + Workshop scientific organizers. These authors contributed equally to this work.
}

Received: 27 February 2020; Accepted: 5 March 2020; Published: 16 March 2020

check for updates

\begin{abstract}
This workshop is the second of the WORKPLANS series, which we started in 2016. The main goal of WORKPLANS is to build up a network of planetary nebulae (PNe) experts to address the main open questions in the field of PNe research. The specific aims of the WORKPLANS workshop series are (i) to discuss and prioritize the most important topics to be investigated by the PN community in the following years; (ii) to establish a network of excellent researchers with complementary expertise; (iii) to formulate ambitious observing proposals for the most advanced telescopes and instrumentation presently available (ALMA, SOFIA, VLT, GTC, HST, etc.), addressing those topics; and (iv) to develop strategies for major proposals to future observatories (JWST, ELT, SPICA, Athena, etc.). To achieve these goals, WORKPLANS II brought together experts in all key sub-areas of the PNe research field, namely: analysis and interpretation of PNe observational data; theoretical modeling of gas and dust emission; evolution from Asymptotic Giant Branch stars (PNe progenitors) to PNe; and the instrumentation and technical characteristics of the relevant observatories.
\end{abstract}

Keywords: planetary nebulae; stars: evolution; stars: late type; stars: mass-loss; stars: winds and outflows; stars: abundances; plasmas; dust; extinction; astrochemistry

\section{Introduction}

Planetary nebulae (PNe) are formed by the ejection of the outer layers of evolved low and intermediate mass stars $\left(\sim 1-8 \mathrm{M}_{\odot}\right)$. These objects have important roles in stellar evolution, in the enrichment of the interstellar medium (ISM) and in the evolution of galaxies. PNe are unique astrophysical laboratories in which we can investigate a wide variety of physical conditions from highly-ionized plasma to low-temperature dusty molecular regions in a spatially resolved manner. The findings from PNe research are applicable not only within the field, but also to a broad range of astrophysical objects with similar characteristics. Solving the open issues in the field of PN would therefore have a great impact not only for the PN research community, but also for the astronomical community as a whole. 
The IAU PN Working Group discusses several open problems in the field of PN in the white paper entitled "The present and future of planetary nebula research" [1]. According to the paper, the main problems to be investigated by the community are:

- uncover PNe that cannot be identified in the optical spectral range

- improve central star evolutionary models

- improve our knowledge of the atmospheres and winds of central stars

- investigate the role of binary central stars in the evolution/formation of PNe

- understand the history and mechanisms of mass-loss and structure formation in the circumstellar nebulae

- improve techniques to derive chemical abundances

- understand the abundance discrepancies measured from collisional and recombination lines

- understand the formation mechanisms and survival of dust and molecules in PNe

The IAU PN working group white paper [1] also makes clear the need of further multi-wavelength observations in the PN research field and serves as a motivation for this workshop series. As in any topic in Astronomy, research on PNe is strongly driven by observations. New facilities built in the last few decades have improved the instruments and opened new spectral windows. Other new and forthcoming facilities, like ALMA and future telescopes such as JWST, will play a key role on the state-of-art astronomy research in the next years. Multiwavelength studies have become possible and, since different wavelength ranges probe different realms of chemistry and physics, the multiwavelength approach has already greatly improved our understanding of a wide range of astronomical objects, including PNe. This provides strong motivation for bringing together researchers with expertise in different wavelength ranges but with a common goal, namely, to understand the formation and evolution of PNe.

To fully explore the possibilities of the current and future instruments for the study of $\mathrm{PNe}$, it is essential to aggregate different expertise-and the formation of an international network of scientists is then a natural step. Such a network provides a framework for the more efficient use of astronomical data and helps the PN community compete for time on the main telescopes, where a strong worldwide presence is an important factor.

The aim of the WORKPLANS series of workshops is to build a network of PN observers whose expertise in observations or theoretical analysis is spread across different sub-fields and spectral domains. The participants are organized in groups to formulate and draft proposal concepts for future observations maximizing the scientific output. During the workshop, we overview the key questions in the field, review the capabilities of observational facilities for (current and future) PN research, discuss which observations are best suited to solving the open problems in the field, and discuss the corresponding necessary analysis (in terms of tools and expertise).

Workshops with similar purposes have been organized with great success by the Asymptotic Giant Branch stars (AGB) research community, which has regular meetings strategically scheduled before each ALMA observing cycle. These meetings generated successful proposals that were granted observing time by this highly competitive observatory. The PNe community is relatively small, with a history of successful collaborations, as exemplified by the ChanPlaNS and HerPlaNS collaborations, both of which have produced significant results (e.g., [2-8]). These surveys were conceived during the Planetary Nebulae Workshop held in Rochester, NY, USA, in 2009 [9], with follow up at the Planetary Nebulae Workshop in Miraflores de la Sierra, Madrid, Spain in 2013; both meetings were organized with goals similar to those of WORKPLANS.

\section{WORKPLANS I}

In the first WORKPLANS (25-29 January 2016), 51 researchers gathered in the Lorentz Center ${ }^{1}$, Leiden, Netherlands, for what was a very fruitful week. We started the meeting with a broad review

1 https://www.lorentzcenter.nl/ 
of potential relevant topics to be investigated in the next years in the PNe field (with talks mainly on Monday). From Tuesday to Thursday, we focused on the scientific and technical aspects of specific wavelength ranges. In the mornings, we reviewed the capabilities of the main telescopes and instruments from each spectral range. The afternoons of these three days were dedicated to group discussions, networking and drafting observing proposals. Brief summaries of those discussion and proposal were given at the end of the day. On Friday, a summary was given by the group leaders, together with the current status of the draft. At least eight proposals were drafted and submitted soon after the workshop.

\section{WORKPLANS II}

The format and goals of WORKPLANS II were similar to its previous edition. The main change was to reduce the time for the "X-ray observations" session, based on experience from WORKPLANS $I$ and the increasing limitations of current facilities. The "X-rays" session was merged into the optical/ultraviolet session, which was made independent from the "infrared observations" session. These changes better balanced the current interests of the PN community and the facilities presently available and under construction. We also made small changes to the program in comparison to WORKPLANS I to add a little more time for plenary discussions. The workshop program is compiled in Table 1.

We organized the workshop to occur with ample time before deadlines for submission of observing proposals for various major facilities (in particular the JWST Cycle 1 proposal deadline), while attempting to avoid periods with classes in universities, to maximize attendance. The workshop was held during the week of 16-20 December 2019, in the Lorentz Center, the Netherlands.

Table 1. WORKPLANS II program.

\begin{tabular}{lll}
\hline Time & Talk & Lecturer/Chair \\
\hline Monday 16 December & \\
\hline 09:30-10:00 & Arrival, office assignment, coffee and tea & \\
10:00-10:10 & Welcome & Lorentz Center Staff \\
10:10-10:20 & Goals and Structure of the Meeting & Isabel Aleman \\
10:20-10:40 & Talk In Honour to Prof. S. Pottasch & Jeronimo Bernard-Salas \\
Session 1: Open Problems and Possible Solutions I & Chair: Letizia Stanghellini \\
10:40-11:05 & Uncertainties in Abundances & Monica Rodriguez \\
11:05-11:30 & The Abundance Discrepancy Problem & Roger Wesson \\
11:30-12:00 & Plenary Discussions & \\
12:00-13:30 & Lunch and Informal Discussions & \\
Session 2: Open Problems and Possible Solutions II & Chair: Jorge Garcia-Rojas \\
13:30-13:55 & S-Process Elements in PNe & Nick Sterling \\
13:55-14:20 & PAHs in PNe & Els Peeters \\
14:20-14:45 & Dust budget in galaxies & Mikako Matsuura \\
14:45-15:10 & Challenges of multi-wavelength PN surveys & Quentin Parker \\
15:10-15:40 & Plenary Discussions & \\
15:40-16:00 & Coffee and Tea Break & \\
Session 3: Open Problems and Possible Solutions III & Chair: Eva Villaver \\
16:00-16:25 & Shaping of Planetary Nebulae & Noam Soker \\
16:25-16:50 & Binaries in Planetary Nebula & David Jones \\
16:50-17:30 & Plenary Discussions & \\
17:30-18:00 & Posters-flash presentations & Wine and Cheese welcoming party with poster session \\
18:00 & & \\
\hline
\end{tabular}


Table 1. Cont

\begin{tabular}{|c|c|c|}
\hline Time & Talk & Lecturer/Chair \\
\hline \multicolumn{3}{|c|}{ Tuesday 17 December } \\
\hline \multicolumn{2}{|c|}{ Session 4: Infrared I } & \multirow{8}{*}{$\begin{array}{l}\text { Chair: Jan Cami } \\
\text { Eric Lagadec } \\
\text { Toshiya Ueta } \\
\text { Raghvendra Sahai } \\
\text { Elvire De Beck }\end{array}$} \\
\hline 09:00-09:30 & IR Observatories & \\
\hline 09:30-09:50 & Observations of Planetary Nebulae with Herschel and AKARI & \\
\hline 09:50-10:10 & Observations of Planetary Nebulae with JWST & \\
\hline 10:10-10:30 & $\begin{array}{l}\text { Observations of PNe/Evolved Stars } \\
\text { with SPICA/Origins Space Telescope }\end{array}$ & \\
\hline 10:30-11:00 & Coffee and tea break & \\
\hline 11:00-12:00 & $\begin{array}{l}\text { Plenary Discussions-Which infrared data do we need } \\
\text { to solve the open questions? }\end{array}$ & \\
\hline $12: 00-13: 30$ & Lunch and Informal Discussions & \\
\hline \multicolumn{2}{|c|}{ Session 5: Infrared II } & \multirow[t]{3}{*}{ Chair: Griet Van de Steene } \\
\hline $13: 30-14: 30$ & Defining Strategic Plan-Defining Projects and Groups & \\
\hline 14:30-18:00 & Hands-on Sessions-Groups discuss and draft proposals & \\
\hline \multicolumn{3}{|c|}{ Wednesday 18 December } \\
\hline \multicolumn{2}{|c|}{ Session 4: Optical, UV, X-Rays I } & \multirow{8}{*}{$\begin{array}{l}\text { Chair: Denise Gor } \\
\text { Romano Corradi } \\
\text { Henri Boffin } \\
\text { Jeremy Walsh } \\
\text { Martin Guerrero }\end{array}$} \\
\hline 09:00-09:30 & Optical/UV Observatories I & \\
\hline 09:30-09:50 & Optical/UV Observatories II & \\
\hline 09:50-10:10 & What MUSE can do for PNe & \\
\hline $10: 10-10: 30$ & X-Rays Observatories and the Observation of PNe & \\
\hline $10: 30-11: 00$ & Coffee and tea break & \\
\hline 11:00-12:00 & $\begin{array}{l}\text { Plenary Discussions-Which Optical/UV/X-rays data } \\
\text { do we need to solve the open questions? }\end{array}$ & \\
\hline 12:00-13:30 & Lunch and Informal Discussions & \\
\hline \multicolumn{2}{|c|}{ Session 5: Optical, UV, X-Rays II } & \multirow[t]{3}{*}{ Chair: Joel Kastner } \\
\hline $13: 30-14: 30$ & Defining Strategic Plan-Defining Projects and Groups & \\
\hline 14:30-18:00 & Hands-on Sessions-Groups discuss and draft proposals & \\
\hline \multicolumn{3}{|c|}{ Thursday 19 December } \\
\hline \multicolumn{2}{|c|}{ Session 6: Radio to Submillimetre I } & \multirow{7}{*}{$\begin{array}{l}\text { Chair: Valentin Bujarrabal } \\
\text { Wouter Vlemmings } \\
\text { Carmen Sanches-Contreras } \\
\text { Laurence Sabin }\end{array}$} \\
\hline 09:00-09:30 & Radio I & \\
\hline 09:30-10:00 & Radio II & \\
\hline 10:00-10:30 & Polarization and Magnetic Fields in PNe & \\
\hline $10: 30-11: 00$ & Coffee and tea break & \\
\hline 11:00-12:00 & $\begin{array}{l}\text { Plenary Discussions-Which radio/Sub/mm data } \\
\text { do we need to solve the open questions? }\end{array}$ & \\
\hline $12: 00-13: 30$ & Lunch and Informal Discussions & \\
\hline \multicolumn{2}{|c|}{ Session 7: Radio to Submillimetre II } & \multirow[t]{3}{*}{ Chair: Albert Zijlstra } \\
\hline $13: 30-14: 30$ & Defining Strategic Plan-Defining Projects and Groups & \\
\hline 14:30-18:00 & Hands-on Sessions-Groups discuss and draft proposals & \\
\hline \multicolumn{3}{|c|}{ Friday 20 December } \\
\hline \multirow{4}{*}{$\begin{array}{l}\text { Session 10: } \\
\text { 09:00-09:30 } \\
\text { 09:30-11:20 } \\
\text { 11:20-12:00 }\end{array}$} & Vrap Up & Chair: Quentin Parker \\
\hline & Workshop Summary & Quentin Parker \\
\hline & Summary of the prepared proposals & Group Leaders \\
\hline & Closing Remarks & Organizers \\
\hline
\end{tabular}

WORKPLANS II had short-term and long-term practical goals. In the short term, we intended to reinforce the previously formed network, inviting at least some of the discussion leaders who emerged during the last workshop while expanding the network by inviting new participants. During the workshop, we formed task forces to address the topics previously mentioned. The task forces focused on a specific spectral realm to strategize proposals designed to use the current forefront facilities that can complement extant data. We invited experts in observations to cover the whole radiative spectrum, from X-rays to radio. Teams were assembled that also included specialists with deep knowledge of the fundamental physics of PNe and hence were prepared to analyze and model the observed objects. 
We also discussed preparations for the use of future instruments, in particular JWST, to be launched in 2022. This represents one of our key long-term goals. Another long-term goal is to keep WORKPLANS as a regular and productive tradition in the PNe community.

Fifty-five participants from all around the world, covering a wide range of expertise, gathered for WORKPLANS II. To maximize the workshop science output, in addition to having a wide range of expertise, in the preparation of our participants list and the program, we also considered the balance of gender, career stage, and geographical representation. The list of participants is compiled in Table 2, and the group picture appears in Figure 1.

Table 2. WORKPLANS II participants list.

\begin{tabular}{|c|c|c|}
\hline Name & Affiliation & Country \\
\hline \multicolumn{3}{|l|}{ Organizers } \\
\hline Isabel Aleman & Universidade Federal de Itajubá & Brazil \\
\hline Jeronimo Bernard-Salas & ACRI-ST & France \\
\hline Joel Kastner & RIT & USA \\
\hline Toshiya Ueta & University of Denver & USA \\
\hline Eva Villaver & Universidad Autónoma de Madrid & Spain \\
\hline \multicolumn{3}{|l|}{ Lecturers and Participants } \\
\hline Stavros Akras & University of Rio Grande & Brazil \\
\hline Karla Ziboney Arellano-Córdova & IAC & Spain \\
\hline Mike Barlow & UCL & UK \\
\hline Henri Boffin & $\mathrm{ESO}$ & Germany \\
\hline Panos Boumis & National Observatory of Athens & Greece \\
\hline Jesse Bublitz & RIT \& IPAG & USA, France \\
\hline Valentin Bujarrabal & OAN & Spain \\
\hline \multirow[t]{2}{*}{ Jan Cami } & University of Western Ontario & Canada \\
\hline & SETI Institute & USA \\
\hline Alessandra Candian & University of Amsterdam & Netherlands \\
\hline Alexandros Chiotellis & National Observatory of Athens & Greece \\
\hline Romano Corradi & GTC - IAC & Spain \\
\hline Elvire De Beck & $\begin{array}{l}\text { Chalmers University of Technology, } \\
\text { Onsala Space Observatory }\end{array}$ & Sweden \\
\hline Jorge Garcia-Rojas & IAC & Spain \\
\hline Veronica Gomez-Llanos & UNAM & Mexico \\
\hline Denise Gonçalves & Valongo Observatory & Brazil \\
\hline Martin Guerrero & IAA-CSIC & Spain \\
\hline Harm Habing & Leiden University & Netherlands \\
\hline David Jones & IAC & Spain \\
\hline Eric Lagadec & Nice Observatory & France \\
\hline Foteini (Claire) Lykou & The University of Hong Kong & China \\
\hline Arturo Manchado & IAC & Spain \\
\hline M. Belén Mari & Valongo Observatory & Brazil \\
\hline Mikako Matsuura & Cardiff University & UK \\
\hline Rodolfo Montez & Harvard-Smithsonian Center for Astrophysics & USA \\
\hline Masaaki Otsuka & Kyoto University & Japan \\
\hline Quentin Parker & The University of Hong Kong & Hong Kong, China \\
\hline \multirow[t]{2}{*}{ Els Peeters } & University of Western Ontario & Canada \\
\hline & SETI Institute & USA \\
\hline Andrés Felipe Perez-Sanchez & Leiden University & Netherlands \\
\hline Guillermo Quintana-lacaci & IFF-CSIC & Spain \\
\hline Matthew Redman & NUI Galway & Ireland \\
\hline Nicole Reindl & University of Potsdam & Germany \\
\hline Andreas Ritter & The University of Hong Kong & Hong Kong, China \\
\hline Mónica Rodríguez & INAOE & Mexico \\
\hline Laurence Sabin & University of Guadalajara & Mexico \\
\hline Raghvendra Sahai & NASA/JPL & USA \\
\hline Carmen Sanches-Contreras & CSIC & Spain \\
\hline Noam Soker & Technion & Israel \\
\hline Letizia Stanghellini & NSF's OIR Lab & USA \\
\hline Nicholas Sterling & University of West Georgia & USA \\
\hline Jesus Toalá & UNAM & Mexico \\
\hline Griet Van de Steene & Royal Observatory & Belgium \\
\hline Wouter Vlemmings & Onsala Space Observatory & Sweden \\
\hline Jeremy Walsh & $\mathrm{ESO}$ & Germany \\
\hline Roger Wesson & UCL & UK \\
\hline Albert Zijlstra & The University of Manchester & UK \\
\hline
\end{tabular}


A good measure of the success of WORKPLANS II was that, by the end of the workshop, the participants were already talking about a third installment.

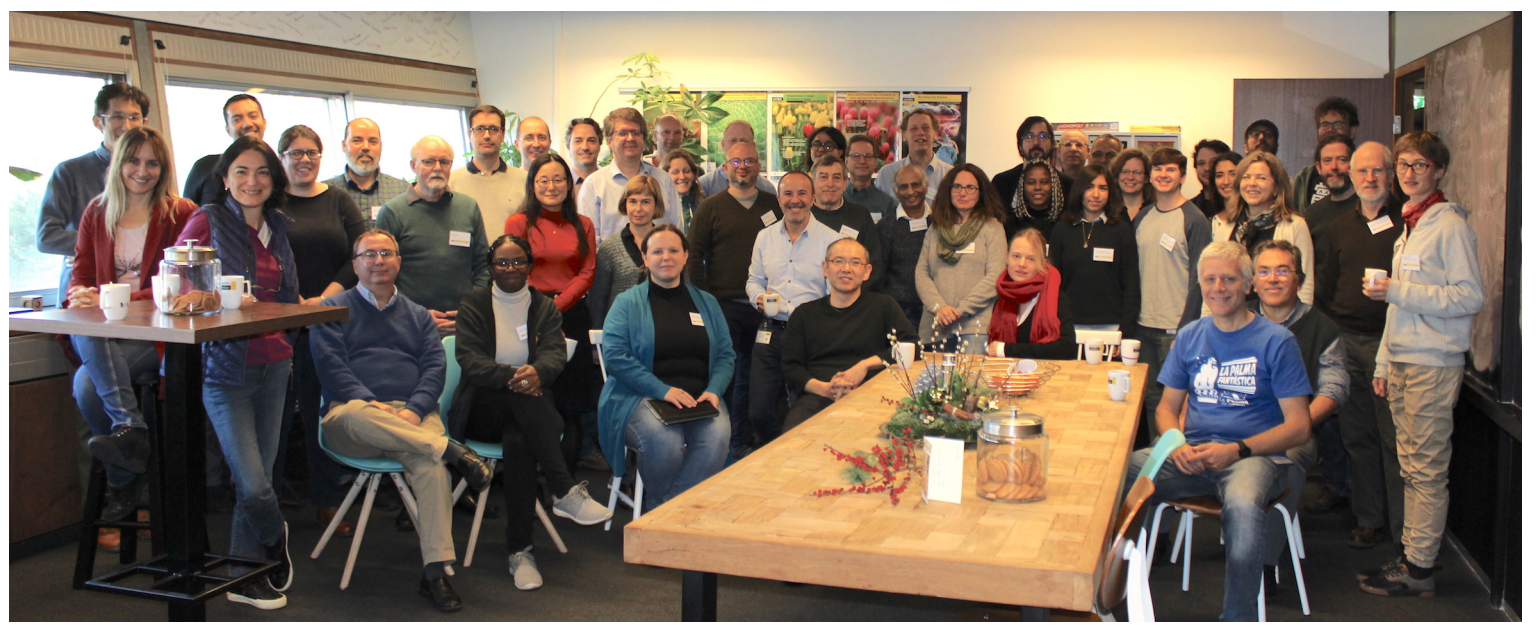

Figure 1. Participants of WORKPLANS II.

\section{Honoring Prof. Stuart Pottasch}

The workshop paid tribute to the life and research of Stuart R. Pottasch and his extensive contribution to Planetary Nebulae research. Stuart was born in 1932 in New York city, and passed away on 4 April 2018 in Groningen. He married Anne Maria de Groot in Leiden with whom he had three children. After she passed away, he then married Greet Mientjes. Besides his passion for Planetary Nebulae, he was also well known for having a large collection of cactuses and parrots. He became one of the youngest professors (if not the youngest) at Groningen University, when he was appointed professor at the Kapteyn Astronomical Institute in 1963 by Adriaan Blaauw. His research legacy includes over 400 papers, of which 93 are first author (43 on PNe), and over 20 Ph.D. students, with his first student being Harm Habing.

While he is known for his research in PNe, Stuart's research is very vast and diverse and includes bright rims, nova outbursts, solar corona, ISM, H II regions, and more. In fact, his two most cited papers are on the interpretation of the Solar UV emission line spectrum. His focus on PNe started with the launch of the Astronomical Netherlands Satellite (ANS) in 1974, and he went on to exploit facilities such as the IUE, IRAS, ISO, Spitzer, and later Herschel to better understand this fascinating stage of stellar evolution. His research in PNe is equally vast, and encompasses nearly all areas of PN research: studies of the central star, nucleosynthesis, nebular physical conditions and chemical composition, dust, PN distances and distribution, and PNe in the galactic context. In 1984, he published a fascinating and very comprehensive book on the subject "Planetary Nebulae: A study of late stages of stellar evolution". During the last two decades, he became very interested in the use of IR lines to derive the nebular chemical composition and their enrichment to the ISM.

While gone, Stuart's research will have a long lasting impact in the study of PNe for many years to come, and a current and new generation of PN scientist will keep benefitting from his tremendous and productive career.

Author Contributions: All authors contributed equally to the workshop and this paper conceptualization, methodology, resources, writing — original draft preparation, writing-review and editing, visualization, and funding acquisition. Project administration and supervision by I.A. All authors have read and agreed to the published version of the manuscript.

Funding: I.A. acknowledges the support of Coordenação de Aperfeiçoamento de Pessoal de Nível Superior-Brasil (CAPES)_Finance Code 001. This research is supported by Space Telescope Science Institute grant HST-GO-15953.001-A to RIT (J.K.). T.U. was supported by NASA under grant NNX15AF24G issued through the Science Mission Directorate. E.V. acknowledges support from the "On the rocks II project" funded by the Spanish Ministerio de Ciencia, Innovación y Universidades under grant PGC2018-101950-B-I00. The 
Workshop was organized with the financial support of the Lorentz Center, which was provided by the Netherlands Organization for Scientific Research (NWO) and the Leiden University.

Acknowledgments: We are very thankful to the Lorentz Center for the organizational support of WORKPLANS I and II.

Conflicts of Interest: The authors declare no conflict of interest.

\section{Abbreviations}

The following abbreviations are used in this manuscript:

$\begin{array}{ll}\text { AGB } & \text { Asymptotic Giant Branch } \\ \text { ALMA } & \text { Atacama Large Millimeter/submillimeter Array } \\ \text { ChanPlaNS } & \text { Chandra Planetary Nebula Survey } \\ \text { CSIC } & \text { Consejo Superior de Investigaciones Científicas } \\ \text { ELT } & \text { Extremely Large Telescope } \\ \text { ESO } & \text { European Southern Observatory } \\ \text { GTC } & \text { Gran Telescopio Canarias } \\ \text { HerPlaNS } & \text { Herschel Planetary Nebula Survey } \\ \text { HST } & \text { Hubble Space Telescope } \\ \text { IAA } & \text { Instituto Astrofísica Andalucía } \\ \text { IAC } & \text { Instituto de Astrofísica de Canarias } \\ \text { IAU } & \text { International Astronomical Union } \\ \text { IFF } & \text { Instituto de Física Fundamental } \\ \text { INAOE } & \text { Instituto Nacional de Astrofísica, Óptica y Electrónica } \\ \text { IPAG } & \text { Institut de Planétologie et d'Astrophysique de Grenoble } \\ \text { ISM } & \text { Interstellar Medium } \\ \text { JPL } & \text { Jet Propulsion Laboratory } \\ \text { JWST } & \text { James Webb Space Telescope } \\ \text { NASA } & \text { National Aeronautics and Space Administration } \\ \text { NSF } & \text { National Science Foundation } \\ \text { NUI } & \text { National University of Ireland } \\ \text { OAN } & \text { Observatorio Astronómico Nacional } \\ \text { OIR Lab } & \text { National Optical-Infrared Astronomy Research Laboratory } \\ \text { PN } & \text { Planetary Nebula } \\ \text { RIT } & \text { Rochester Institute of Technology } \\ \text { SETI } & \text { Search for Extraterrestrial Intelligence } \\ \text { SOFIA } & \text { Stratospheric Observatory for Infrared Astronomy } \\ \text { SPICA } & \text { Space Infrared Telescope for Cosmology and Astrophysics } \\ \text { UCL } & \text { University College London } \\ \text { UNAM } & \text { Universidad Nacional Autónoma de Mexico } \\ \text { VLT } & \text { Very Large Telescope } \\ \text { WORKPLANS } & \text { Workshop on Planetary Nebula Observations } \\ & \end{array}$

\section{References}

1. Kwitter, K.B.; Méndez, R.H.; Peña, M.; Stanghellini, L.; Corradi, R.L.M.; De Marco, O.; Fang, X.; Henry, R.B.C.; Karakas, A.I.; Liu, X.W.; et al. The Present and Future of Planetary Nebula Research. A White Paper by the IAU Planetary Nebula Working Group. Revista Mexicana de AstronomíA y AstrofíSica 2014, 50, $203-223$.

2. Kastner, J.H.; Montez, R., Jr.; Balick, B.; Frew, D.J.; Miszalski, B.; Sahai, R.; Blackman, E.; Chu, Y.H.; De Marco, O.; Frank, A.; et al. The Chandra X-Ray Survey of Planetary Nebulae (ChanPlaNS): Probing Binarity, Magnetic Fields, and Wind Collisions. Astron. J. 2012, 144, 58. [CrossRef]

3. Freeman, M.; Montez, R., Jr.; Kastner, J.H.; Balick, B.; Frew, D.J.; Jones, D.; Miszalski, B.; Sahai, R.; Blackman, E.; Chu, Y.H.; et al. The Chandra Planetary Nebula Survey (ChanPlaNS). II. X-ray Emission from Compact Planetary Nebulae. Astrophys. J. 2014, 794, 99. [CrossRef] 
4. Ueta, T.; Ladjal, D.; Exter, K.M.; Otsuka, M.; Szczerba, R.; Siódmiak, N.; Aleman, I.; van Hoof, P.A.M.; Kastner, J.H.; Montez, R.; et al. The Herschel Planetary Nebula Survey (HerPlaNS). I. Data overview and analysis demonstration with NGC 6781. Astron. Astrophys. 2014, 565, A36. [CrossRef]

5. Aleman, I.; Ueta, T.; Ladjal, D.; Exter, K.M.; Kastner, J.H.; Montez, R.; Tielens, A.G.G.M.; Chu, Y.H.; Izumiura, H.; McDonald, I.; et al. Herschel Planetary Nebula Survey (HerPlaNS). First, detection of $\mathrm{OH}^{+}$in planetary nebulae. Astron. Astrophys. 2014, 566, A79. [CrossRef]

6. Montez, R., Jr.; Kastner, J.H.; Balick, B.; Behar, E.; Blackman, E.; Bujarrabal, V.; Chu, Y.H.; Corradi, R.L.M.; De Marco, O.; Frank, A.; et al. The Chandra Planetary Nebula Survey (ChanPlaNS). III. X-Ray Emission from the Central Stars of Planetary Nebulae. Astrophys. J. 2015, 800, 8. [CrossRef]

7. Otsuka, M.; Ueta, T.; van Hoof, P.A.M.; Sahai, R.; Aleman, I.; Zijlstra, A.A.; Chu, Y.H.; Villaver, E.; Leal-Ferreira, M.L.; Kastner, J.; et al. The Herschel Planetary Nebula Survey (HerPlaNS): A Comprehensive Dusty Photoionization Model of NGC6781. Astrophys. J. 2017, 231, 22. [CrossRef]

8. Aleman, I.; Exter, K.; Ueta, T.; Walton, S.; Tielens, A.G.G.M.; Zijlstra, A.; Montez, R.; Abraham, Z.; Otsuka, M.; Beaklini, P.P.B.; et al. Herschel Planetary Nebula Survey (HerPlaNS). Hydrogen recombination laser lines in Mz 3. Mon. Not. R. Astron. Soc. 2018, 477, 4499-4510. [CrossRef]

9. De Marco, O.; Frank, A.; Kastner, J.; Sahai, R.; Balick, B.; Blackman, E.; Carroll, J.; Chesneau, O.; Chu, Y.H.; Claussen, M.; et al. The Rochester White Paper: A Roadmap for Understanding Aspherical Planetary Nebulae. In Proceedings of the Asymmetric Planetary Nebulae 5 Conference, Bowness-on-Windermere, UK, 20-25 June 2010; p. 19.

(C) 2020 by the authors. Licensee MDPI, Basel, Switzerland. This article is an open access article distributed under the terms and conditions of the Creative Commons Attribution (CC BY) license (http:// creativecommons.org/licenses/by/4.0/). 